\title{
ON THE RELATIVE DE RHAM SEQUENCE
}

\author{
N. BUCHDAHL
}

\begin{abstract}
The classical de Rham sequence on a (smooth, paracompact) manifold provides a connection between solutions of certain differential equations and the topology of the manifold. This paper shows how the relative de Rham sequence for a mapping between manifolds gives a connection between solutions of differential equations and the topology of the fibres of the mapping.
\end{abstract}

The classical de Rham sequence on a (smooth) manifold provides a connection between solutions of certain differential equations and the topology of the manifold; this note shows how the relative de Rham sequence for a mapping between manifolds gives a connection between solutions of differential equations and the topology of the fibres of the mapping.

The notation used here is as follows: a resolution of a sheaf $\mathcal{S}$ on a space $X$ by sheaves $\Re^{p}, p=0,1, \ldots$, is abbreviated by $0 \rightarrow \mathcal{S} \rightarrow \Re$, the de Rham cohomology of the resolution by $H^{*}\left(\Gamma\left(X, \Re^{*}\right)\right)$, and the topological inverse image under a map $f$ : $Y \rightarrow X$ by $f^{-1} \delta$. The symbols $\vartheta$ and $\xi$ are generic for the holomorphic and smooth categories respectively; here "smooth" can be interpreted as either real- or complexvalued: the symbol $k$ will be used to denote either $\mathbf{R}$ or $\mathbf{C}$.

If $X, Y$ are smooth manifolds and $f: Y \rightarrow X$ is a smooth mapping, the sheaf of germs of relative 1 -forms $\xi_{f}^{1}$ on $Y$ is defined by the exact sequence $f^{*} \xi_{X}^{1} \rightarrow \xi_{Y}^{1} \rightarrow \xi_{f}^{1} \rightarrow$ 0 , and relative $p$-forms by $\xi_{f}^{p}:=\Lambda^{p} \xi_{f}^{1}$. (Relative forms may be thought of as forms along the fibres of $f$ parametrized by the variables transverse to the fibres.) The induced mapping $\xi_{f}^{p} \rightarrow \xi_{f}^{p+1}$, (differentiation along the fibres), is denoted by $d_{f}$, and when $f$ is of maximal rank there is a resolution on $Y, 0 \rightarrow f^{-1} \xi_{X} \rightarrow \xi_{f}$, the relative de Rham sequence. (An analogous relative de Rham sequence exists for the holomorphic category, but this will not be considered here.) Since the resolution is acyclic, there are canonical isomorphisms $H^{p}\left(Y, f^{-1} \xi_{X}\right) \simeq H^{p}\left(\Gamma\left(Y, \xi_{f}\right)\right)$ which will be used without comment.

Proposition 1. Let $X, Y$ be ( paracompact) smooth manifolds, and $f: Y \rightarrow X$ be a surjective smooth mapping of maximal rank. Then if $H^{p-1}\left(f^{-1}(x), k\right)=$ $H^{p}\left(f^{-1}(x), k\right)=0$ for all $x \in X$ and some $p>0$ (where, for $p=1$ the former topological condition is taken to mean $f^{-1}(x)$ is connected), it follows that $H^{p}\left(Y, f^{-1} \xi_{X}\right)=0$.

Received by the editors March 16, 1982.

1980 Mathematics Subject Classification. Primary 58A12. 
Proof. Let $x_{0} \in X$ and $K \subset \subset Y$ be fixed. By a theorem by Weil [3] there exists a simple covering of $f^{-1}\left(x_{0}\right)$; i.e. a locally finite open covering by relatively compact open sets such that every intersection in the nerve of the covering is contractible. By considering singular homology with coefficients in $k$ on $f^{-1}\left(x_{0}\right)$, the hypothesis $H^{p}\left(f^{-1}\left(x_{0}\right), k\right)=0$ easily implies the existence of open subsets $U, V$ of $f^{-1}\left(x_{0}\right)$ with $f^{-1}\left(x_{0}\right) \cap K \subset \subset U \subset \subset V \subset \subset f^{-1}\left(x_{0}\right)$ and which are finite unions of open sets in a simple covering, such that $H^{p}(V, k) \rightarrow H^{p}(U, k)$ is the zero map.

Using standard differential-geometric techniques, one can find a small neighbourhood $W$ of $x_{0}$ such that $V \times W$ is diffeomorphic to neighbourhood of $\bar{U}$ in $Y$, the induced mapping $\tilde{f}: V \times W \rightarrow X$ being projection onto second factor. (See any proof of the local triviality of a smooth family of compact manifolds.)

The finite covering of $V \times W$ by sets of the form $S \times W$, where $S$ is one of the sets in a simple covering chosen originally to define $V$, forms a Leray cover for the sheaf $\tilde{f}^{-1} \xi_{X}$. If $(c(x))$ is a $p$-cocycle with coefficients in $\tilde{f}^{-1} \xi_{X}$ on $V \times W$ with respect to this covering, then the finite system of equations determined by $\delta b=c$ is compatible on $U \times W$ since the conclusion of the first paragraph implies that the system $\delta b(x)=c(x)$ has a solution on $U$ for each fixed $x$ in $W$.

Thus $H^{p}\left(V \times W, \tilde{f}^{-1} \xi_{X}\right) \rightarrow H^{p}\left(U \times W, \tilde{f}^{-1} \xi_{X}\right)$ is the zero map, and by utilizing the isomorphism $H^{*}\left(f^{-1} \xi_{X}\right)=H^{*}\left(\Gamma\left(\xi_{f}^{*}\right)\right)$, a simple partition of unity argument enables one to deduce the existence of open sets $U^{\prime}, V^{\prime}$ with $K \subset \subset U^{\prime} \subset \subset V^{\prime} \subset \subset Y$ such that $H^{p}\left(V^{\prime}, f^{-1} \xi_{X}\right) \rightarrow H^{p}\left(U^{\prime}, f^{-1} \xi_{X}\right)$ is the zero map.

Hence one can find an exhaustion $\left\{Y_{\nu}: \nu=1,2, \ldots\right\}$ of $Y$ by open sets $Y_{\nu}$ with $Y_{\nu} \subset \subset Y_{\nu+1}$ such that

(*) $\quad H^{q}\left(Y_{\nu+1}, f^{-1} \xi_{X}\right) \rightarrow H^{q}\left(Y_{\nu}, f^{-1} \xi_{X}\right)$ is the zero map for $q=p-1, p$.

(For $p=1$, the former condition is replaced by the connectivity of $Y_{\nu}$.) Given now a $d_{f}$-closed relative $p$-form $g$ on $Y,{ }^{*}(p)$ implies the existence of solutions to $d_{f} u_{\nu}=g$ on each $U_{\nu}$, whilst ${ }^{*}(p-1)$ enables one to inductively modify the sequence $\left\{u_{\nu}\right\}$ (using suitable cut-off functions) to obtain a convergent sequence whose limit $u \in \Gamma\left(Y, \xi_{f}^{p-1}\right)$ satisfies $d_{f} u=g$ everywhere on $Y$. Q.E.D.

Remark. When $Y$ is a product $Y=X \times F$ with $f$ being projection, the proof is easily modified to dispense with the condition $H^{p^{-1}}\left(f^{-1}(x), k\right)=0$, as might be expected. In general, however, this is not possible as the following example demonstrates: $Y=\mathbf{R}^{2} \backslash$ origin $, \quad X=\mathbf{R}, f:(x, y) \mapsto x$. Then the relative 1 -form $x\left(x^{2}+y^{2}\right)^{-1} d y$ is not $d$ fexact. Similarly, higher dimensional counterexamples are easily constructed.

In a recent paper of Eastwood, Penrose and Wells [1], the authors exhibit a process whereby cohomology with values in certain holomorphic vector bundles on a particular complex manifold is transformed into solutions of certain (holomorphic) differential equations on a second complex manifold-a kind of cohomological Radon transform. In the concluding section of that paper, the comment is made that the technique can clearly be applied to far more general situations, and it is this comment which motivates the following corollary providing sufficient conditions under which a general such transform will be invertible. 
COROllary. Let $X, Y$ be complex manifolds, $f: Y \rightarrow X$ be a surjective holomorphic mapping of maximal rank, and $B$ be a holomorphic vector bundle on $X$. If, for some $N \geqslant 0, H^{p}\left(f^{-1}(x), \mathbf{C}\right)=0$ for $p=0,1, \ldots, N$ and all $x \in X$, then the canonical homomorphism $H^{q}(X, \vartheta(B)) \rightarrow H^{q}\left(Y, f^{-1} \vartheta(B)\right)$ is an isomorphism for $q=0,1, \ldots, N$, and a monomorphism for $q=N+1$.

Proof. The Dolbeault resolution on $X, 0 \rightarrow \vartheta(B) \rightarrow \xi^{0 \cdot}(B)$, pulls back to a resolution on $Y, 0 \rightarrow f^{-1} \vartheta(B) \rightarrow f^{-1} \xi^{0 \cdot}(B)$. A partition of unity argument together with the proposition leads to the conclusion that $H^{q}\left(Y, f^{-1} \xi^{0 \cdot} \cdot(B)\right)=0$ for $q=$ $1,2, \ldots, N$, from which the result follows immediately. Q.E.D.

There is a partial converse to Proposition 1, which rests on the usual Fréchet space structure given to spaces of forms and relative forms. The proof uses the facts that (a) a sequence of continuous linear mappings $E \rightarrow^{u} F \rightarrow^{v} G$ between Fréchet spaces is exact if the (topological) dual sequence $E^{\prime} \leftarrow{ }^{\prime} F^{\prime} \leftarrow{ }^{v^{\prime}} G^{\prime}$ is and $u(E)$ is closed in $F$, and (b) the dual sequence is exact if $E \rightarrow F \rightarrow G$ is and $v(F)$ is closed in $G$.

Proposition 2. Let $X, Y$ and $f$ be as in Proposition 1, and suppose that for some $p \geqslant 0, H^{p}\left(Y, f^{-1} \xi_{X}\right)=H^{p+1}\left(Y, f^{-1} \xi_{X}\right)=0$, (where, for $p=0$, the former condition is replaced by " $\Gamma\left(X, \xi_{X}\right) \rightarrow \Gamma\left(Y, f^{-1} \xi_{X}\right)$ is an isomorphism"). Then $H^{p}\left(f^{-1}(x), k\right)=$ 0 for all $x \in X$. (For $p=0$, this means $f^{-1}(x)$ is connected, as before.)

Proof. Fix $x_{0} \in X$, and endow $\Gamma\left(Y, \xi_{f}^{*}\right), \Gamma\left(f^{-1}\left(x_{0}\right), \xi^{*}\right)$ with families of seminorms $\left\{\|\cdot\|_{K, \nu}: \nu=1,2, \ldots ; K\right.$ compact with supp $\left.\|\cdot\|_{K, \nu} \subset K\right\}$ such that the induced topology on these spaces is the usual Fréchet topology.

To prove the result, it must be shown that $\Gamma\left(f^{-1}\left(x_{0}\right), \xi^{p-1}\right) \rightarrow^{d} \Gamma\left(f^{-1}\left(x_{0}\right), \xi^{p}\right)$ $\rightarrow^{d} \Gamma\left(f^{-1}\left(x_{0}\right), \xi^{p+1}\right)$ is exact; (here $\Gamma\left(f^{-1}\left(x_{0}\right), \xi^{-1}\right):=k$ when $\left.p=0\right)$. However, it is a standard result that $d \Gamma\left(\xi^{q}\right)$ is always closed in $\Gamma\left(\xi^{q+1}\right)$, (see e.g. Hörmander [2, p. 187]), so by remark (a) above, it suffices to demonstrate the exactness of the dual sequence. This will now be shown to be a consequence of the exactness of $\Gamma\left(Y, \xi_{f}^{p-1}\right)^{\prime} \leftarrow \Gamma\left(Y, \xi_{f}^{p}\right)^{\prime} \leftarrow \Gamma\left(Y, \xi_{f}^{p+1}\right)^{\prime}$, which itself follows from the hypotheses and (b) above; (when $p=0, \Gamma\left(Y, \xi_{f}^{-1}\right):=\Gamma\left(X, \xi_{X}\right)$ ).

So suppose that $A \in \Gamma\left(f^{-1}\left(x_{0}\right), \xi^{p}\right)^{\prime}$ satisfies $d^{\prime} A=0$. Define $\tilde{A} \in \Gamma\left(Y, \xi_{f}^{p}\right)^{\prime}$ by $\tilde{A}(g):=A\left(g\left(x_{0}\right)\right)$. Then $d_{f}^{\prime} \tilde{A}=0$, so there exists $\tilde{B} \in \Gamma\left(Y, \xi^{p+1}\right)^{\prime}$ with $d_{f}^{\prime} \tilde{B}=\tilde{A}$. Since $A$ and $\tilde{B}$ are continuous, there are compact sets $L \subset f^{-1}\left(x_{0}\right)$ and $K \subset Y$ such that $|A| \leqslant$ const $\|\cdot\|_{L, \mu}$ and $|B| \leqslant$ const $\|\cdot\|_{K, \nu}$, and one may assume without loss of generality that $L \subset f^{-1}\left(x_{0}\right) \cap K$. As in the proof of Proposition 1, one can find a neighbourhood $W$ of $x_{0}$ and open sets $U, V \subset \subset f^{-1}\left(x_{0}\right)$ with $f^{-1}\left(x_{0}\right) \cap K \subset U$ $\subset \subset V$ and $V \times W$ diffeomorphic to a neighbourhood $V^{\prime}$ of $\bar{U}$ in $Y$, such that $f^{-1}(W) \cap K \subset U^{\prime} \quad\left(:=\right.$ image of $U \times W$ under $\left.V \times W \simeq V^{\prime}\right)$. Choosing $\chi \in$ $C_{0}^{\infty}(W), \psi \in C_{0}^{\infty}(V)$ such that $\chi\left(x_{0}\right)=\left.1 \equiv \psi\right|_{\bar{U}}$, one can define a linear functional $B$ on $\Gamma\left(f^{-1}\left(x_{0}\right), \xi^{p+1}\right)$ by $B(g):=\tilde{B}(\psi \chi g)$, (in the obvious sense: the splitting $\left.\left.\left.\xi_{Y}^{l}\right|_{V^{\prime}} \simeq \xi_{f^{-1}\left(x_{0}\right)}^{1}\right|_{V} \oplus \xi_{X}^{1}\right|_{W}$ is being used implicitly here).

It is now a simple matter to check that $B$ is continuous and that it satisfies $d^{\prime} B=A$. Q.E.D.

Note that the same example as given in the remark following Proposition 1 can be used to show that the hypothesis $H^{p+1}\left(Y, f^{-1} \xi_{X}\right)=0$ is not redundant in general. 
The author wishes to thank Dr. M. G. Eastwood for his assistance in the preparation of this manuscript, and the Royal Commission for the Exhibition of 1851 for financial support during the period of its writing.

\section{REFERENCES}

I. M. (j. Eastwood, R. Penrose and R. O. Wells, Jr., Cohomology and massless fields. Comm. Math. Phys. 78 (1981), 305-351.

2. L. Hörmander, An introduction of complex analysis in several variables, North-Holland, Amsterdam and London, 1973.

3. A. Weil, Sur les théoremes de de Rham, Comment. Math. Helv. 26 (1952), 119-145.

DePARTMENT OF MATHEMATICS, UNIVERSITY OF OXFORD, OXFORD OXI 2JD, UNITED Kingdom

Current address: Institut des Hautes Études Scientifiques, 91440 Bures-sur-Yvette, France 